\title{
An adaptive stabilized finite element scheme for the advection-reaction-diffusion equation
}

\author{
Rodolfo Araya $^{1}$, Edwin Behrens ${ }^{2}$, and Rodolfo Rodríguez ${ }^{3}$ \\ $\mathrm{GI}^{2} \mathrm{MA}$, Departamento de Ingeniería Matemática, Universidad de Concepción, \\ Casilla 160-C, Concepción, Chile.
}

\begin{abstract}
An adaptive finite element scheme for the advection-reaction-diffusion equation is introduced and analyzed. This scheme is based on a stabilized finite element method combined with a residual error estimator. The estimator is proved to be reliable and efficient. More precisely, global upper and local lower error estimates with constants depending at most on the local mesh Peclet number are proved. The effectiveness of this approach is illustrated by several numerical experiments.
\end{abstract}

Key words: Advection-reaction-diffusion problem, boundary and inner layers, $a$ posteriori error estimates, stabilized finite elements.

\section{Introduction}

This paper deals with the advection-diffusion-reaction equation. This kind of problems arise in many application, for instance, when linearizing the NavierStokes problem, to model pollutant transport and degradation in aquatic media, etc. In particular, our work is motivated by the need of an efficient scheme to be used in a water quality model for the Bío Bío River in Chile.

Specially interesting is the case when advective or reactive terms are dominant. In this case, the solution of the equation frequently has exponential or parabolic boundary layers (for details see [9]). The standard Galerkin approximation usually fails in this situation because this method introduces

1 Partially supported by FONDECYT 1040595 (Chile).

2 Supported by CONICYT (Chile).

${ }^{3}$ Partially supported by FONDAP in Applied Mathematics (Chile). 
nonphysical oscillations. A possible remedy is to add to the variational formulation some numerical diffusion terms to stabilize the finite element solution. Some examples of this approach are the streamline upwind Petrov-Galerkin method (SUPG) (see [3]), the Galerkin least squares approximation (GLS) (see [6]), the Douglas-Wang method (see [5]), the 'unusual' stabilized finite element method (USFEM) (see [7]), and the residual-free bubbles approximation (RFB) (see [2]). The drawback with most of these methods is that the solution layers are not very well resolved, because of the numerical diffusion added to the discretization.

There are not so many references in the literature dealing with a posteriori techniques for this equation. The reason of this is that most of the standard error estimators involve equivalence constants depending on negative powers of the diffusion parameter, which lead to very poor results in the advective or reactive dominated cases. An error estimator which is robust in the sense of leading to global upper and local lower bounds depending at most on the local mesh Peclet number has been developed by Verfürth (see [12] and [13]). Using these results Sangalli has analyzed a residual a posteriori error estimate for the residual-free bubbles scheme (see [10]). On the other hand, Knopp et al. have developed some a posteriori error estimates using a stabilized scheme combined with a shock-capturing technique to control the local oscillations in the crosswind direction (see [8]). Finally, Wang has introduced an error estimate for the advection-diffusion equation based on the solution of local problems on each element of the triangulation (see [15]).

In this paper we introduce and analyze from theoretical and experimental points of view an adaptive scheme to efficiently solve the advection-reactiondiffusion equation. This scheme is based on the stabilized finite element method introduced in [7] combined with an error estimator similar to the one developed in [13]. We prove global upper and local lower error estimates in the energy norm, with constants which only depend on the shape-regularity of the mesh, the polynomial degree of the finite element approximating space and the local mesh Peclet number. We perform several numerical experiments to show the effectiveness of our approach to capture boundary and inner layers very sharply and without significant oscillations. The experiments also show that the scheme attains optimal order of convergence.

The paper is organized as follows. In Section 2 we recall the advection-diffusionreaction problem under consideration and the stabilized scheme. In Section 3 we define an a posteriori error estimator and prove its equivalence with the energy norm of the finite element approximation error. Finally, in Section 4, we introduce the adaptive scheme and report the results of the numerical tests. 


\section{A stabilized method for a model problem}

Our model problem is the advection-reaction-diffusion equation

$$
\left\{\begin{aligned}
-\varepsilon \Delta u+\boldsymbol{a} \cdot \nabla u+b u & =f & & \text { in } \Omega \\
u & =0 & & \text { on } \Gamma_{\mathrm{D}} \\
\varepsilon \frac{\partial u}{\partial \boldsymbol{n}} & =g & & \text { on } \Gamma_{\mathrm{N}}
\end{aligned}\right.
$$

where $\Omega \subset \mathbb{R}^{2}$ is a bounded polygonal domain with a Lipschitz boundary $\Gamma=\bar{\Gamma}_{\mathrm{D}} \cup \bar{\Gamma}_{\mathrm{N}}$, with $\Gamma_{\mathrm{D}} \cap \Gamma_{\mathrm{N}}=\emptyset$. We denote by $\boldsymbol{n}$ the outer unit normal vector to $\Gamma$.

We are interested in the advection-reaction dominated case and assume that:

(A1) $\varepsilon \in \mathbb{R}: 0<\varepsilon \ll 1$;

$(\mathrm{A} 2) \boldsymbol{a} \in \mathrm{W}^{1, \infty}(\Omega)^{2}, \quad b \in \mathrm{L}^{\infty}(\Omega), \quad\|\boldsymbol{a}\|_{\infty, \Omega}+\|b\|_{\infty, \Omega}=\mathcal{O}(1)$;

(A3) $\operatorname{div} \boldsymbol{a}=0, \quad b \geq 1$;

(A4) $\Gamma_{\mathrm{D}} \supset\{\boldsymbol{x} \in \Gamma: \boldsymbol{a}(\boldsymbol{x}) \cdot \boldsymbol{n}(\boldsymbol{x})<0\}$;

(A5) $f \in \mathrm{L}^{2}(\Omega), g \in \mathrm{L}^{2}\left(\Gamma_{\mathrm{N}}\right)$.

We use standard notation for Sobolev and Lebesgue spaces and norms. Moreover, let $\mathrm{H}_{\Gamma_{\mathrm{D}}}^{1}(\Omega):=\left\{\varphi \in \mathrm{H}^{1}(\Omega):\left.\varphi\right|_{\Gamma_{\mathrm{D}}}=0\right\}$ and $B$ be the bilinear form defined on $\mathrm{H}^{1}(\Omega)$ by

$$
B(v, w):=\int_{\Omega}(\varepsilon \nabla v \cdot \nabla w+\boldsymbol{a} \cdot \nabla v w+b v w) .
$$

Then, the standard variational formulation of problem (2.1) is the following: Find $u \in \mathrm{H}_{\Gamma_{\mathrm{D}}}^{1}(\Omega)$ such that

$$
B(u, v)=\int_{\Omega} f v+\int_{\Gamma_{\mathrm{N}}} g v \quad \forall v \in \mathrm{H}_{\Gamma_{\mathrm{D}}}^{1}(\Omega)
$$

We consider the (energy) norm $\|u\| \|:=\left(\varepsilon\|\nabla u\|_{0, \Omega}^{2}+\|u\|_{0, \Omega}^{2}\right)^{\frac{1}{2}}$ defined on $\mathrm{H}^{1}(\Omega)$. Assumptions (A1)-(A4) and integration by parts imply that

$$
B(v, v) \geq\|v\|^{2} \quad \forall v \in \mathrm{H}_{\Gamma_{\mathrm{D}}}^{1}(\Omega)
$$

and

$$
B(v, w) \leq\left(1+\|b\|_{\infty, \Omega}+\varepsilon^{-\frac{1}{2}}\|\boldsymbol{a}\|_{\infty, \Omega}\right)\|\| v\|\|\|w\| .
$$

Hence, as a consequence of Lax-Milgram's Lemma, problem (2.3) has a unique solution. 
Let $\left\{\mathcal{T}_{h}\right\}_{h>0}$, be a family of shape-regular partitions of $\Omega$ into triangles. Let $V_{h}:=\left\{\varphi \in \mathrm{H}_{\Gamma_{\mathrm{D}}}^{1}(\Omega):\left.\varphi\right|_{T} \in \mathcal{P}_{k} \forall T \in \mathcal{T}_{h}\right\}$, where, for $k \in \mathbb{N}, \mathcal{P}_{k}$ denotes the space of polynomials of degree at most $k$. It is well known that the standard Galerkin method with this finite element space yields poor approximation when $\varepsilon \ll|\boldsymbol{a}|$ or $\varepsilon \ll b$. For this reason we consider the following stabilized formulation introduced in [7]: Find $u_{h} \in V_{h}$ such that

$$
B_{\tau}\left(u_{h}, v_{h}\right)=F_{\tau}\left(v_{h}\right) \quad \forall v_{h} \in V_{h}
$$

where, for $v_{h}, w_{h} \in V_{h}$,

$$
\begin{aligned}
& B_{\tau}\left(v_{h}, w_{h}\right):=B\left(v_{h}, w_{h}\right) \\
& -\sum_{T \in \mathcal{T}_{h}} \int_{T} \tau_{T}\left(-\varepsilon \Delta v_{h}+\boldsymbol{a} \cdot \nabla v_{h}+b v_{h}\right)\left(-\varepsilon \Delta w_{h}-\boldsymbol{a} \cdot \nabla w_{h}+b w_{h}\right)
\end{aligned}
$$

and

$$
F_{\tau}\left(v_{h}\right):=\int_{\Omega} f v_{h}+\int_{\Gamma_{\mathrm{N}}} g v_{h}-\sum_{T \in \mathcal{T}_{h}} \int_{T} \tau_{T} f\left(-\varepsilon \Delta v_{h}-\boldsymbol{a} \cdot \nabla v_{h}+b v_{h}\right) .
$$

In the expressions above we use a stabilization parameter $\tau_{T}$ defined as follows:

$$
\tau_{T}(\boldsymbol{x}):=\frac{h_{T}^{2}}{b(\boldsymbol{x}) h_{T}^{2} \max \left\{1, \mathrm{Pe}_{T}^{\mathrm{R}}(\boldsymbol{x})\right\}+\left(2 \varepsilon / m_{k}\right) \max \left\{1, \mathrm{Pe}_{T}^{\mathrm{A}}(\boldsymbol{x})\right\}},
$$

where $\mathrm{Pe}_{T}^{\mathrm{R}}(\boldsymbol{x})$ and $\mathrm{Pe}_{T}^{\mathrm{A}}(\boldsymbol{x})$ are respectively defined by

$$
\mathrm{Pe}_{T}^{\mathrm{R}}(\boldsymbol{x}):=\frac{2 \varepsilon}{m_{k} b(\boldsymbol{x}) h_{T}^{2}} \quad \text { and } \quad \mathrm{Pe}_{T}^{\mathrm{A}}(\boldsymbol{x}):=\frac{m_{k}|\boldsymbol{a}(\boldsymbol{x})| h_{T}}{\varepsilon},
$$

where

$$
m_{k}:=\min \left\{1 / 3, C_{k}\right\}
$$

with $C_{k}$ being a positive constant satisfying

$$
C_{k} \sum_{T \in \mathcal{T}_{h}} h_{T}^{2}\left\|\Delta v_{h}\right\|_{0, T}^{2} \leq\left\|\nabla v_{h}\right\|_{0, \Omega}^{2} \quad \forall v_{h} \in V_{h}
$$

which only depends on the polynomial degree $k$ and the shape-regularity of the mesh.

Finally $h_{T}$ is a measure of the element size. Under the presence of advection $(\boldsymbol{a} \neq \mathbf{0})$, it is reported in [7] that an element parameter $h_{T}$ which yields very good numerical results is the largest streamline distance in the element, as shown in Fig. 1. As can be seen in this figure, to compute $h_{T}$, we take the velocity constant on the element: $\boldsymbol{a}_{T}:=\boldsymbol{a}\left(\boldsymbol{x}_{T}\right)$, with $\boldsymbol{x}_{T}$ being the barycenter of $T$. If $\boldsymbol{a}_{T}=\mathbf{0}$, we take $h_{T}$ equal to the diameter of $T$. 


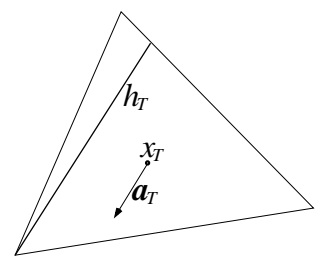

Fig. 1. Element parameter $h_{T}$.

The following lemma shows that the bilinear form $B_{\tau}$ is positive definite and, consequently, the stabilized discrete problem (2.5) is well posed. This has been proved in Lemma 1 of [7] under the assumptions that the coefficients of the advection-diffusion-reaction equation (2.1) are constant and the boundary conditions are purely Dirichlet. It is straightforward to extend the same arguments to our case to prove the following result.

Lemma 1 Under assumptions (A1)-(A4),

$$
B_{\tau}\left(v_{h}, v_{h}\right) \geq \sum_{T \in \mathcal{T}_{h}}\left[\int_{T} \varepsilon \beta_{T}\left|\nabla v_{h}\right|^{2}+\int_{T} \tau_{T}\left(\boldsymbol{a} \cdot \nabla v_{h}\right)^{2}+\int_{T} b \beta_{T}\left|v_{h}\right|^{2}\right]
$$

for all $v_{h} \in V_{h}$, with $\beta_{T}:=\frac{\varepsilon}{m_{k} b h_{T}^{2}+2 \varepsilon}>0, T \in \mathcal{T}_{h}$.

The convergence and stabilization properties of this scheme have also been investigated in [7], where numerical experiments proving the effectiveness of this approach have been reported. However, these experiments also show that the method does not allow a sharp resolution of inner layers when quasi-uniform meshes are used. In the following section we introduce an error indicator which will allow us to create in an automatic fashion meshes correctly refined around inner and boundary layers of the solution.

\section{A posteriori error estimator.}

In this section we define a residual error estimator, similar to one analyzed in [13] in the context of advection-reaction-diffusion.

Let $\mathcal{E}_{h}$ denote the set of all edges in $\mathcal{T}_{h}$ and, for $E \in \mathcal{E}_{h}$, let $h_{E}$ be the length of $E$. For each mesh $\mathcal{T}_{h}$, let $f_{h} \in V_{h}$ and $g_{h} \in\left\{\left.v_{h}\right|_{\Gamma_{\mathrm{N}}}: v_{h} \in V_{h}\right\}$ be arbitrary but fixed approximations of $f$ and $g$, respectively. We define the approximate volumetric and edge residuals by

$$
R_{T}^{h}\left(u_{h}\right):=f_{h}+\varepsilon \Delta u_{h}-a \cdot \nabla u_{h}-b u_{h}, \quad T \in \mathcal{T}_{h},
$$




$$
R_{E}^{h}\left(u_{h}\right):= \begin{cases}-\llbracket \varepsilon \frac{\partial u_{h}}{\partial \boldsymbol{n}_{E}} \rrbracket_{E}, & \text { if } E \in \mathcal{E}_{h}: E \nsubseteq \Gamma, \\ g_{h}-\varepsilon \frac{\partial u_{h}}{\partial \boldsymbol{n}}, & \text { if } E \in \mathcal{E}_{h}: E \subset \Gamma_{\mathrm{N}}, \\ 0, & \text { if } E \in \mathcal{E}_{h}: E \subset \Gamma_{\mathrm{D}} .\end{cases}
$$

These residuals are used to define an estimator of the local error in energy norm, $\left\|u-u_{h}\right\|_{T}^{2}:=\varepsilon\left\|\nabla\left(u-u_{h}\right)\right\|_{0, T}^{2}+\left\|u-u_{h}\right\|_{0, T}^{2}$, as follows

$\eta_{T}^{2}:=\alpha_{T}^{2}\left\|R_{T}^{h}\left(u_{h}\right)\right\|_{0, T}^{2}+\frac{1}{2} \sum_{E \subset \partial T \cap \Omega} \varepsilon^{-\frac{1}{2}} \alpha_{E}\left\|R_{E}^{h}\left(u_{h}\right)\right\|_{0, E}^{2}+\sum_{E \subset \partial T \cap \Gamma_{\mathrm{N}}} \varepsilon^{-\frac{1}{2}} \alpha_{E}\left\|R_{E}^{h}\left(u_{h}\right)\right\|_{0, E}^{2}$,

with

$$
\alpha_{S}:=\min \left\{h_{S} \varepsilon^{-\frac{1}{2}}, 1\right\}, \quad S \in \mathcal{T}_{h} \cup \mathcal{E}_{h} .
$$

Let us recall that $h_{T}$ is not the diameter of $T$, but the largest streamline distance in the element (see Fig. 1). However, $h_{T}$ is equivalent to the diameter of $T$ with equivalence constants only depending on the element shape ratio.

The efficiency and reliability of a similar estimator applied to a standard SUPG method has been proved in [13]. In what follows we show that analogous results hold for our estimator applied to the stabilized method described in the previous section. To this aim we first prove the following technical lemmas.

Lemma 2 Given $T \in \mathcal{T}_{h}$, let $\tau_{T}$ be defined by (2.8). Then the following bounds hold $\forall \boldsymbol{x} \in T$ :

$$
\varepsilon \tau_{T}(\boldsymbol{x}) \leq \frac{1}{6} h_{T}^{2}, \quad|\boldsymbol{a}(\boldsymbol{x})| \tau_{T}(\boldsymbol{x}) \leq \frac{1}{2} h_{T}, \quad b(\boldsymbol{x}) \tau_{T}(\boldsymbol{x}) \leq 1
$$

Furthermore,

$$
b(\boldsymbol{x}) \tau_{T}(\boldsymbol{x}) \leq C \alpha_{T}, \quad \text { with } C:=\max \left\{1,\left(\|b\|_{\infty, \Omega} / 6\right)^{\frac{1}{2}}\right\}
$$

PROOF. For the first estimate, we use (2.8) and (2.9) to obtain

$$
\varepsilon \tau_{T}(\boldsymbol{x}) \leq \frac{\varepsilon h_{T}^{2}}{b(\boldsymbol{x}) h_{T}^{2} \max \left\{1, \mathrm{Pe}_{T}^{\mathrm{R}}(\boldsymbol{x})\right\}} \leq \frac{\varepsilon h_{T}^{2}}{b(\boldsymbol{x}) h_{T}^{2} \mathrm{Pe}_{T}^{\mathrm{R}}(\boldsymbol{x})} \leq \frac{m_{k}}{2} h_{T}^{2} \leq \frac{1}{6} h_{T}^{2} .
$$

For the second one, if $\boldsymbol{a}(\boldsymbol{x})=\mathbf{0}$ there is nothing to prove; otherwise, by using (2.8) and (2.9) we have

$$
|\boldsymbol{a}(\boldsymbol{x})| \tau_{T}(\boldsymbol{x}) \leq \frac{|\boldsymbol{a}(\boldsymbol{x})| m_{k} h_{T}^{2}}{2 \varepsilon \max \left\{1, \mathrm{Pe}_{T}^{\mathrm{A}}(\boldsymbol{x})\right\}} \leq \frac{|\boldsymbol{a}(\boldsymbol{x})| m_{k} h_{T}^{2}}{2 \varepsilon \mathrm{Pe}_{T}^{\mathrm{A}}(\boldsymbol{x})} \leq \frac{1}{2} h_{T} .
$$


For the third bound, from (2.8),

$$
b(\boldsymbol{x}) \tau_{T}(\boldsymbol{x}) \leq \frac{1}{\max \left\{1, \mathrm{Pe}_{T}^{\mathrm{R}}(\boldsymbol{x})\right\}} \leq 1 .
$$

Moreover, from the first estimate of this lemma, $b(\boldsymbol{x}) \tau_{T}(\boldsymbol{x}) \leq b(\boldsymbol{x}) h_{T}^{2} /(6 \varepsilon)$, too. Hence, taking the geometric mean of this and the third estimate we have

$$
b(\boldsymbol{x}) \tau_{T}(\boldsymbol{x}) \leq\left(\frac{\|b\|_{\infty, \Omega}}{6}\right)^{\frac{1}{2}} h_{T} \varepsilon^{-\frac{1}{2}}
$$

From this and the third estimate again, we conclude the last inequality.

Here and thereafter, $C$ denotes a generic positive constant, not necessarily the same at each occurrence, but always independent of the mesh-size and the small parameter $\varepsilon$.

Lemma 3 The following estimates hold for all $w_{h} \in V_{h}$ :

$$
\left\|\nabla w_{h}\right\|_{0, T} \leq C h_{T}^{-1} \alpha_{T}\left\|w_{h}\right\|_{T} \quad \text { and } \quad\left\|\Delta w_{h}\right\|_{0, T} \leq C h_{T}^{-2} \alpha_{T}\left\|w_{h}\right\|_{T} .
$$

PROOF. The definition of the energy norm $\||\cdot|||$ implies

$$
\left\|\nabla w_{h}\right\|_{0, T} \leq \varepsilon^{-\frac{1}{2}}\left\|w_{h}\right\|_{T}
$$

whereas, from a standard scaling argument,

$$
\left\|\nabla w_{h}\right\|_{0, T} \leq C h_{T}^{-1}\left\|w_{h}\right\|_{0, T} \leq C h_{T}^{-1}\left\|w_{h}\right\|_{T},
$$

with the constant $C$ only depending on the shape ratio of the element $T$ and the polynomial degree $k$ in the definition of the finite element space $V_{h}$. Then, from these two inequalities, we obtain the first estimate.

On the other hand, another scaling argument and (3.5) yield

$$
\left\|\Delta w_{h}\right\|_{0, T} \leq C h_{T}^{-1}\left\|\nabla w_{h}\right\|_{0, T} \leq C h_{T}^{-1} \varepsilon^{-\frac{1}{2}}\left\|w_{h}\right\|_{T},
$$

whereas, using scaling arguments again, we have

$$
\left\|\Delta w_{h}\right\|_{0, T} \leq C h_{T}^{-2}\left\|w_{h}\right\|_{T} .
$$

Finally, we obtain the second estimate from these last two inequalities. 
In what follows we will show that the energy norm of the error can be bounded by means of the estimators $\eta_{T}$. To do this, first we write from (2.4)

$$
\left\|u-u_{h}\right\| \leq \sup _{v \in \mathrm{H}_{\Gamma_{\mathrm{D}}}^{1}(\Omega) \backslash\{0\}} \frac{B\left(u-u_{h}, v\right)}{\|v\|} .
$$

Second, let $I_{h}: L^{2}(\Omega) \longrightarrow V_{h}$ be the Clément interpolation operator (see [4]). Several estimates in energy norm for this operator have been proved in Lemma 3.2 of [13]. In particular, it has been shown that for all $T \in \mathcal{T}_{h}$ and $v \in \mathrm{H}^{1}\left(\widetilde{\omega}_{T}\right)$, with $\widetilde{\omega}_{T}:=\bigcup\left\{\bar{T}^{\prime} \in \mathcal{T}_{h}: \bar{T}^{\prime} \cap \bar{T} \neq \emptyset\right\}$, there holds

$$
\left\|\left|I _ { h } v \left\|_{T} \leq C\left|\|v \mid\|_{\widetilde{\omega}_{T}}\right.\right.\right.\right.
$$

Now, consider an arbitrary $v \in \mathrm{H}_{\Gamma_{\mathrm{D}}}^{1}(\Omega)$ with $\|v\| \|=1$. Obviously, we have

$$
B\left(u-u_{h}, v\right)=B\left(u-u_{h}, v-I_{h} v\right)+B\left(u-u_{h}, I_{h} v\right) .
$$

To estimate this terms we introduce the exact volumetric and edge residuals $R_{T}\left(u_{h}\right)$ and $R_{E}\left(u_{h}\right)$, which are defined as in (3.1) and (3.2), but with $f_{h}$ and $g_{h}$ substituted by $f$ and $g$, respectively. For the first term in the right hand side above, we have the following estimate,

$$
B\left(u-u_{h}, v-I_{h} v\right) \leq C\left[\sum_{T \in \mathcal{T}_{h}} \alpha_{T}^{2}\left\|R_{T}\left(u_{h}\right)\right\|_{0, T}^{2}+\sum_{E \in \mathcal{E}_{h}} \varepsilon^{-\frac{1}{2}} \alpha_{E}\left\|R_{E}\left(u_{h}\right)\right\|_{0, E}^{2}\right]^{\frac{1}{2}},
$$

which has been proved in [13] (see equation (4.5)) with $h_{T}$ being the diameter of $T$. However the same arguments are valid in our case.

For the second term in the right hand side of (3.7) we have the following estimate.

Lemma 4 For all $v \in \mathrm{H}_{\Gamma_{\mathrm{D}}}^{1}(\Omega)$ with $\|v\| \|=1$,

$$
B\left(u-u_{h}, I_{h} v\right) \leq C\left[\sum_{T \in \mathcal{T}_{h}} \alpha_{T}^{2}\left\|R_{T}\left(u_{h}\right)\right\|_{0, T}^{2}\right]^{\frac{1}{2}}
$$

PROOF. For all $w_{h} \in V_{h}$, from (2.2), (2.3), (2.5), (2.6), and (2.7), we have

$$
B\left(u-u_{h}, w_{h}\right)=-\sum_{T \in \mathcal{T}_{h}} \int_{T} \tau_{T} R_{T}\left(u_{h}\right)\left(-\varepsilon \Delta w_{h}-a \cdot \nabla w_{h}+b w_{h}\right) .
$$


Next, from Lemmas 2 and 3, straightforward computations lead to

$$
\int_{T} \tau_{T} R_{T}\left(u_{h}\right)\left(-\varepsilon \Delta w_{h}-a \cdot \nabla w_{h}+b w_{h}\right) \leq C \alpha_{T}\left\|R_{T}\left(u_{h}\right)\right\|_{0, T}\left\|w_{h}\right\|_{T} .
$$

Finally, we replace $w_{h}$ by $I_{h} v$ and use (3.6) to obtain

$$
B\left(u-u_{h}, I_{h} v\right) \leq C \sum_{T \in \mathcal{T}_{h}} \alpha_{T}\left\|R_{T}\left(u_{h}\right)\right\|_{0, T}\|v\| \|_{\widetilde{\omega}_{T}}
$$

Thus, the lemma follows from the regularity of the mesh.

Now we are able to state the main theoretical result of this paper.

Theorem 5 Let $u$ and $u_{h}$ be the solutions of problems (2.3) and (2.5), respectively. Let $f_{h}$ and $g_{h}$ be arbitrary approximations of $f$ and $g$ by elements of $V_{h}$ and traces on $\Gamma_{\mathrm{N}}$ of elements of $V_{h}$, respectively. Let $\eta_{T}$ be defined by (3.1)-(3.4). Then, there holds

$$
\begin{aligned}
\left\|u-u_{h}\right\| \leq C & {\left[\left(\sum_{T \in \mathcal{T}_{h}} \eta_{T}^{2}\right)^{\frac{1}{2}}\right.} \\
& \left.+\left(\sum_{T \in \mathcal{T}_{h}} \alpha_{T}^{2}\left\|f-f_{h}\right\|_{0, T}^{2}+\sum_{E \subset \Gamma_{\mathrm{N}}} \varepsilon^{-\frac{1}{2}} \alpha_{E}\left\|g-g_{h}\right\|_{0, E}^{2}\right)^{\frac{1}{2}}\right]
\end{aligned}
$$

and

$$
\begin{aligned}
\eta_{T} \leq C & \left(1+\|b\|_{\infty, \omega_{T}}+\varepsilon^{-\frac{1}{2}}\|\boldsymbol{a}\|_{\infty, \omega_{T}} \alpha_{T}\right)\left\|u-u_{h}\right\|_{\omega_{T}} \\
& +\alpha_{T}\left\|f-f_{h}\right\|_{0, \omega_{T}}+\left(\sum_{E \subset \partial T \cap \Gamma_{\mathrm{N}}} \varepsilon^{-\frac{1}{2}} \alpha_{E}\left\|g-g_{h}\right\|_{0, E}^{2}\right)^{\frac{1}{2}} \quad \forall T \in \mathcal{T}_{h},
\end{aligned}
$$

where $\omega_{T}:=\bigcup\left\{\bar{T}^{\prime} \in \mathcal{T}_{h}: \bar{T}^{\prime} \cap \bar{T} \supset E \in \mathcal{E}_{h}\right\}$.

PROOF. The first estimate is a consequence of (3.7), (3.8), Lemma 4, and the definition of the estimator $\eta_{T}$. The second estimate can be proved by following similar techniques to those used in Proposition 4.1 of [13]. 


\section{Numerical experiments}

In this section we report three numerical experiments with the stabilized method described in Section 2 and an $h$-adaptive mesh-refinement strategy based on the error estimator analyzed in Section 3. In all the experiments we have used piecewise linear finite elements and we have taken as geometric domain the unit square $\Omega:=(0,1) \times(0,1)$, although with different boundary conditions. We have considered varying values of the coefficients of the advection-reaction-diffusion equation (2.1).

The adaptive procedure consists in solving problem (2.5) on a sequence of meshes up to finally attain a solution with an estimated error within a prescribed tolerance. To attain this purpose, we initiate the process with a quasiuniform mesh and, at each step, a new mesh better adapted to the solution of problem (2.3) must be created. This is done by computing the local error estimators $\eta_{T}$ for all $T$ in the 'old' mesh $\mathcal{T}_{h}$, and refining those elements $T$ with $\eta_{T} \geq \theta \max \left\{\eta_{T}: T \in \mathcal{T}_{h}\right\}$, where $\theta \in(0,1)$ is a prescribed parameter. In all our experiments we have chosen $\theta=\frac{1}{2}$.

We have used a Matlab code adapted by us from [1] and the mesh generator Triangle. This generator allows us to create successively refined meshes based on a hybrid Delaunay refinement algorithm (see [11]).

\subsection{A reaction-diffusion problem}

The first test consists in solving a purely reaction-diffusion problem. We have chosen the following data: $\boldsymbol{a}=\mathbf{0}, b=1, f=1$, the boundary conditions shown in Fig. 2, and various values of the parameter $\varepsilon$. The exact solution of this problem is $u(x, y)=1-\sinh \left(\varepsilon^{-1 / 2} x\right) / \sinh \left(\varepsilon^{-1 / 2}\right)$.

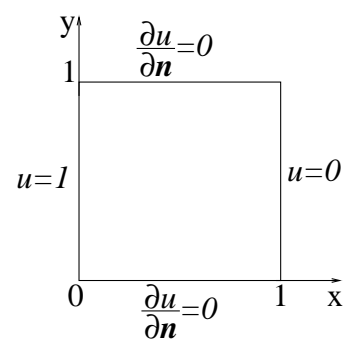

Fig. 2. Boundary conditions for the reaction-diffusion problem.

Fig. 3 shows some of the successively refined meshes created in the adaptive process for $\varepsilon=10^{-4}$. This figure also shows the horizontal cuts at $y=0.5$ of the corresponding computed solution. The iteration number and the number 
of degrees of freedom (d.o.f.) of each mesh are also reported in this figure. Fig. 4 shows the error curves of the whole process for the exact and estimated errors. This figure also includes a line with slope $-\frac{1}{2}$, which corresponds to the theoretically optimal order of convergence for piecewise linear elements.
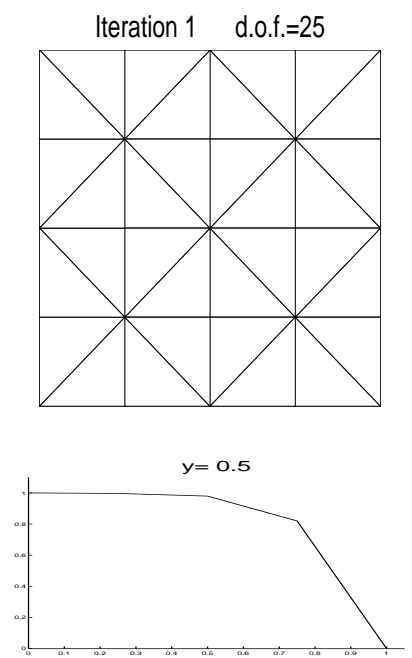

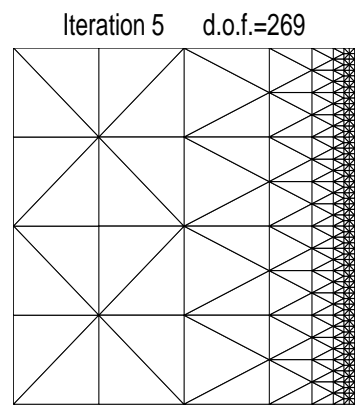

$y=0.5$

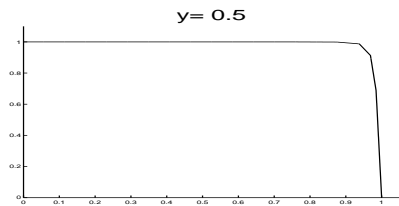

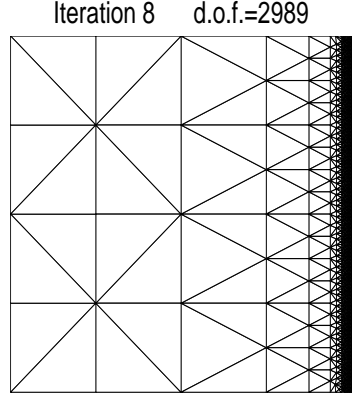

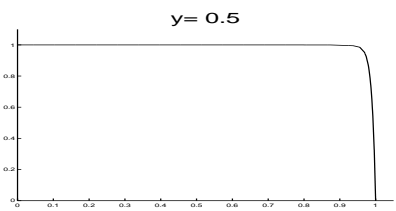

Fig. 3. Reaction-diffusion problem. Meshes and horizontal cuts of the approximate solutions.

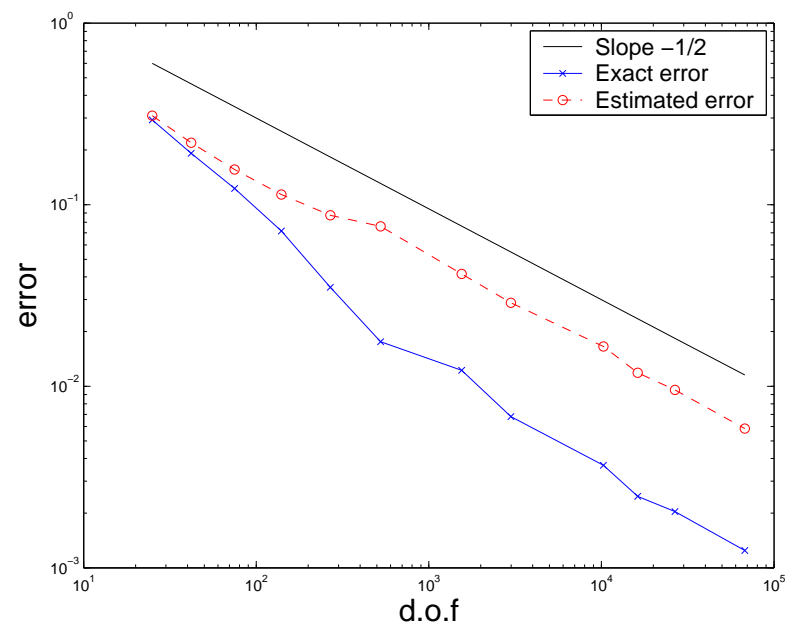

Fig. 4. Reaction-diffusion problem. Estimated and exact error curves.

It can be seen from Fig. 3 that the adaptive process leads to meshes correctly refined in the boundary layer zone. The adaptive scheme is able to capture the boundary layer very sharply and without any significant oscillation.

On the other hand, the error curves show that the adaptive process yields optimal order convergence. This happens in spite of the fact that the effectivity indices are very poor. Indeed, it can be observed in Fig. 4 that the exact error is severely overestimated. Anyway, the exact and estimated error curves have approximately the same optimal slope $-\frac{1}{2}$. 


\subsection{An advection-diffusion problem}

The second test consists in solving a purely advection-diffusion problem. We have chosen the following data: $\boldsymbol{a}=(1,0), b=0, f=1$, and the boundary conditions shown in Fig. 5. In this case, the solution of this problem is $u(x, y)=x-\left(\mathrm{e}^{-\frac{1-x}{\varepsilon}}-\mathrm{e}^{-\frac{1}{\varepsilon}}\right) /\left(1-\mathrm{e}^{-\frac{1}{\varepsilon}}\right)$. Let us remark that the chosen value of $b$ formally violates assumption (A3).

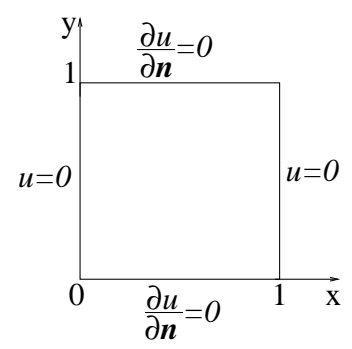

Fig. 5. Boundary conditions for the advection-diffusion problem.

We report again the results obtained for $\varepsilon=10^{-4}$. Fig. 6 shows some of the successively refined meshes created in the adaptive process and the horizontal cuts at $y=0.5$ of the corresponding computed solution. Fig. 7 shows the error curves for the exact and estimated errors.
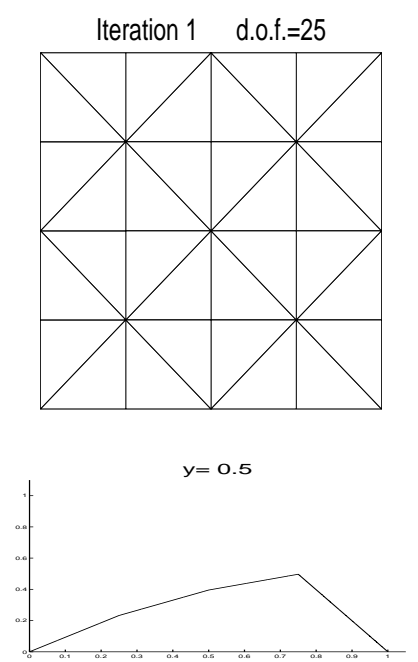
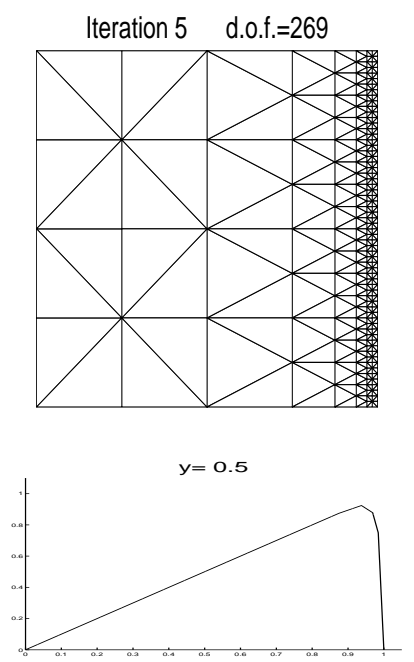
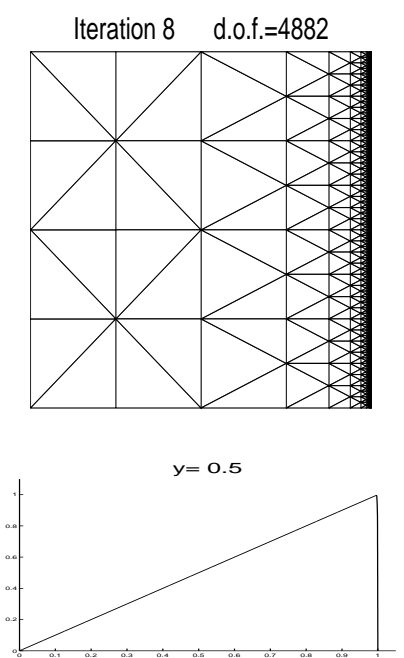

Fig. 6. Advection-diffusion problem. Meshes and horizontal cuts of the approximate solutions.

Essentially the same conclusions as in the previous test can be drawn from Fig. 6: the boundary layers are very sharply captured without any significant oscillation. On the other hand, the error curves in Fig. 7 attain almost optimal slopes $-\frac{1}{2}$, once the meshes are sufficiently refined around the singular zone of the solutions. 


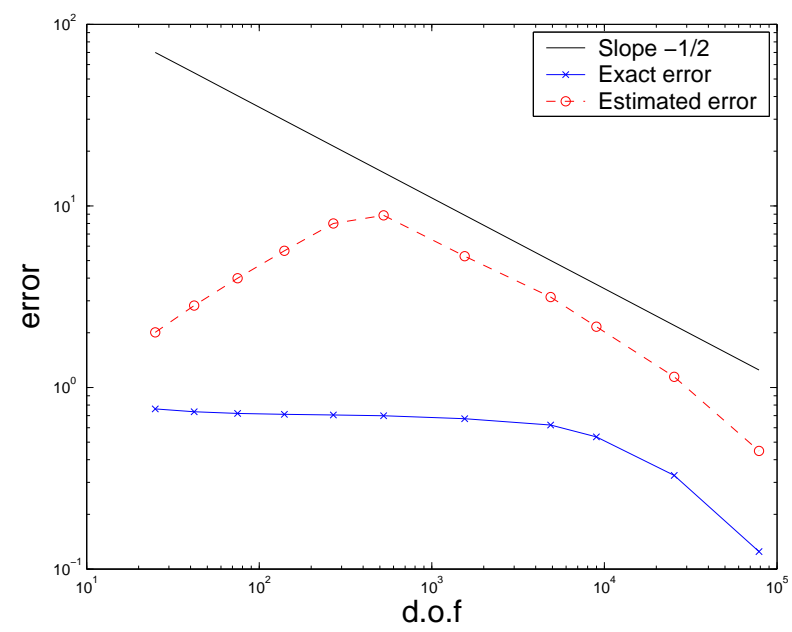

Fig. 7. Advection-diffusion problem. Estimated and exact error curves.

\subsection{An advection-diffusion-reaction problem with an inner layer}

The last reported test consists in solving an advection-diffusion-reaction problem whose solution presents an inner layer. We have chosen the same example considered in [13] (Problem $N$ in this reference). The corresponding data are: $\boldsymbol{a}=(2,1), b=1, f=0$, and the boundary conditions are shown in Fig. 8. Let us remark that this problem is not covered by our theoretical results, since the chosen Dirichlet boundary condition have a jump at the origin; hence, the problem cannot have a solution in $\mathrm{H}^{1}(\Omega)$. We do not include error curves because no analytical solution is known in this case.

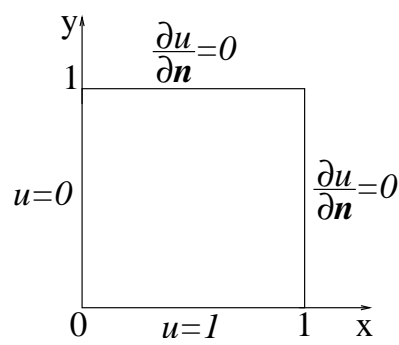

Fig. 8. Boundary conditions for the advection-reaction-diffusion problem.

Once more, we report the results obtained for $\varepsilon=10^{-4}$. Fig. 9 shows some of the successively refined meshes created in the adaptive process, as well as the level sets of the corresponding computed solution. The latter shows that the oscillations are completely eliminated in the final meshes. 

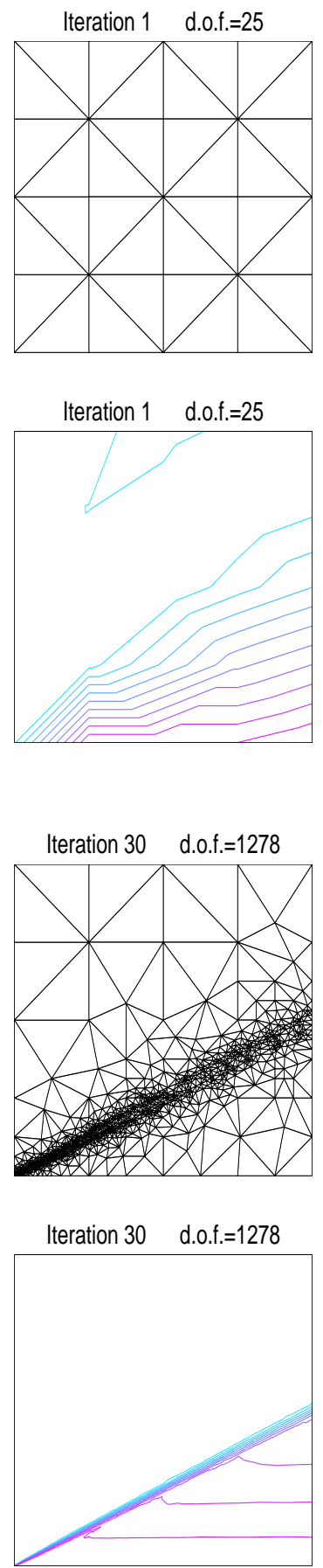
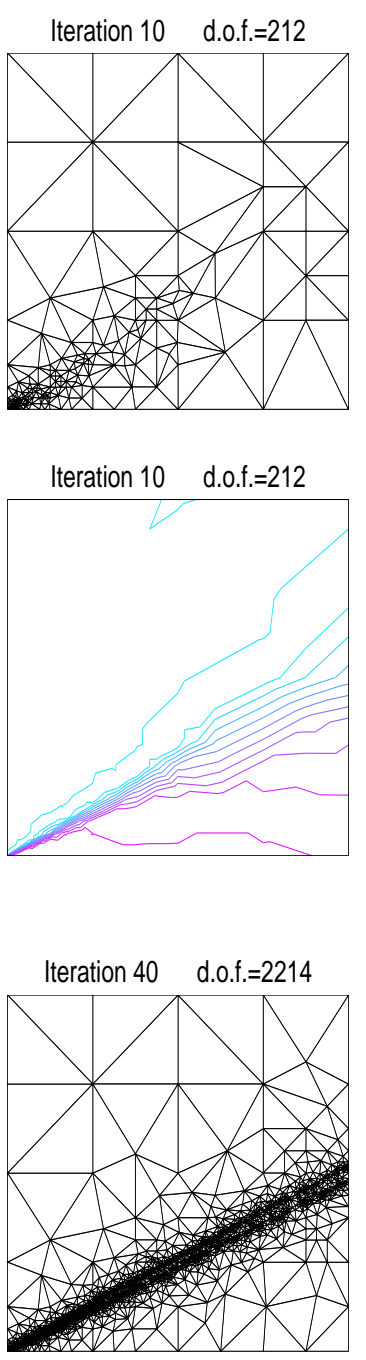

$$
\text { Iteration } 40 \quad \text { d.o.f. }=2214
$$

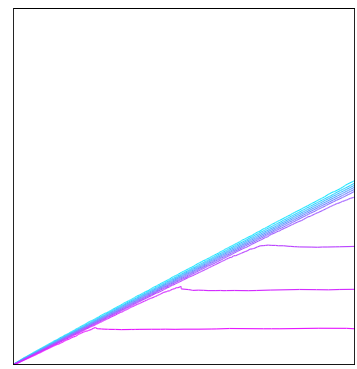

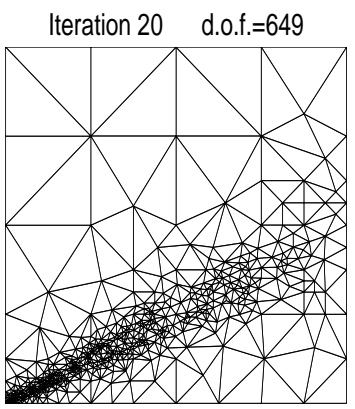
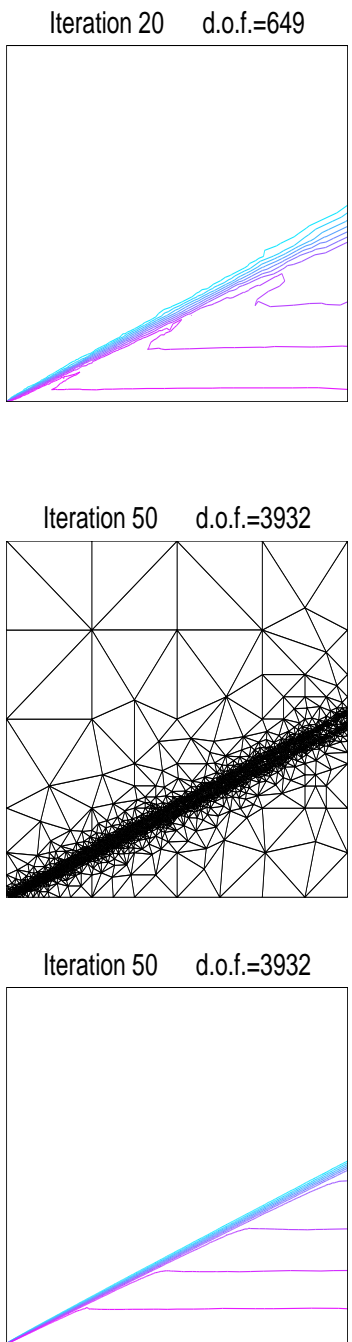

Fig. 9. Advection-diffusion-reaction problem. Meshes and level sets.

These figures show clearly that the adaptive process leads once more to correctly refined meshes. In both cases the adaptive scheme detects both, the corner singularity of the solution and the inner layer, and creates meshes much more concentrated around these zones. Once the corner singularity is resolved, the adaptive scheme detects the inner layer and advance through it refining the mesh. Finally, the method allows us to capture the inner layer very sharply and without any significant oscillation. 


\section{Conclusions}

An adaptive finite element scheme for the advection-reaction-diffusion equation has been introduced and analyzed. This scheme is based on a stabilized finite element method combined with a residual error estimator. The estima-

tor is shown to be reliable and efficient in that global upper and local lower error estimates are rigorously proved.

Several numerical experiments are reported. All of them show the effectiveness of this scheme to capture boundary and inner layers very sharply and without significant oscillations. Furthermore, the experiments show that the scheme attains optimal order of convergence.

\section{Acknowledgements}

This work was done in the framework of FONDEF research project D001135.

The authors want to thank Gabriel Barrenechea by helpful discussions and suggestions, as well as the anonymous referees.

\section{References}

[1] J. Alberty, C. Carstensen, S.A. Funken, Remarks around 50 lines of Matlab: short finite element implementation, Numer. Algor. 20 (1999) 117-137.

[2] F. Brezzi, D. Marini, A. Russo, Applications of the pseudo residual-free bubbles to the stabilization of convection-diffusion problems, Comput. Methods Appl. Mech. Engrg. 166 (1998) 51-63.

[3] A.N. Brooks, T.J.R. Hughes, Streamline upwind/Petrov-Galerkin formulations for convection dominated flows with particular emphasis on the incompressible Navier-Stokes equations, Comput. Methods Appl. Mech. Engrg. 32 (1982) 199259.

[4] P. Clément, Approximation by finite element functions using local regularization, RAIRO, R-2 (1975) 77-84.

[5] J. Douglas, J.P. Wang, An absolutely stabilized finite element method for the Stokes problem, Math. Comp. 52 (1989) 495-508.

[6] L.P. Franca, S.L. Frey, T.J.R. Hughes, Stabilized finite element methods. I. Application to the advective-diffusive model, Comput. Methods Appl. Mech. Engrg. 95 (1992) 253-276. 
[7] L.P. Franca, F. Valentin, On an improved unusual stabilized finite element method for the advective-reactive-diffusive equation, Comput. Methods Appl. Mech. Engrg. 190 (2000) 1785-1800.

[8] T. Knopp, G. Lube, G. Rapin, Stabilized finite element methods with shock capturing for advection-diffusion problems, Comput. Methods Appl. Mech. Engrg. 191 (2002) 2997-3013.

[9] H.-G. Roos, M. Stynes, L. Tobiska, Numerical Methods for Singularly Perturbed Differential Equations - Convection-Diffusion and Flow Problems, Springer Series in Computational Mathematics, Vol. 24, Springer-Verlag, 1996.

[10] G. Sangalli, A robust a posteriori estimator for the residual-free bubbles method applied to advection-diffusion problems, Numer. Math. 89 (2001) 379-399.

[11] J.R. Shewchuk, Delaunay refinement algorithms for triangular mesh generation, Comp. Geom. Theor. Appl. 22 (2002) 21-74.

[12] R. Verfürth, Robust a posteriori error estimators for a singularly perturbed reaction-diffusion equation, Numer. Math. 78 (1998) 479-493.

[13] R. Verfürth, A posteriori error estimators for convection-diffusion equations, Numer. Math. 80 (1998) 641-663.

[14] R. Verfürth, A Review of A Posteriori Error Estimation and Adaptive Meshrefinement Techniques Wiley-Teubner, Chichester, Stuttgart, 1996.

[15] S. Wang, An a posteriori error estimate for finite element approximations of a singularly perturbed advection-diffusion problem, J. Comput. Appl. Math. 87 (1997) 227-242. 